\title{
EFECTOS DE LA CONSEJERÍA PERSONALIZADA EN LA REDUCCIÓN DE CIFRAS TENCIONALES EN PACIENTES HIPERTENSOS ESENCIALES SEVEROS, OMS I
}

\author{
Jasna Stiepovich Bertoni* \\ Tatiana Paravic Klijn** \\ Carmen Garfe Carreño*** \\ Herman Diaz Bugueño****
}

Se compararon dos sistemas de intervención para evaluar el efecto de la consejería personalizada en pacientes hipertensos esenciales severos, OMS I, orientados a reducir cifras tensionales sistólicas, diastólicas y aumentar su autoestima. La variable independente como método de intervención tuvo una duración de 24 semanas. Se midieron variables despendientes: cifras tensionales y autoestima. Los resultados arrojaron qué pacientes hipertensos esenciales severos OMS I, del grupo experimental disminuyeron en promedio más sus cifras tensionales y aumentaron en promedio más su autoestima que pacientes del grupo control, encontrándose también depencia entre peso y presión arterial. Consejería Personalizada demostró ser una estrategia de Enfermería exitosa, para este grupo de pacientes.

UNITERMOS: Consejería personalizada, paciente hipertenso

\footnotetext{
*Facultad de Medicina, Departamento de Enfermería, Universidad de Concepción. Profesor Asociado. Directora Departamento de Enfermería. Magíster en Enfermería con mención en Enfermería Médico-Quirúrgica.

**Facultad de Medicina, Departamento de Enfermería, Universidad de Concepción. Profesor Asociado. Magíster en Enfermería con Mención en Enfermería en Salud Comunitaria.

***Hospital las Higueras Talcahuano. Servicio de Salud Talcahuano. Médico Cirujano, Especialista en Medicina Interna.

****Hospital las Higueras Talcahuano. Servicio de Salud Talcahuano. Médico Cirujano, Especialista en Medicina Interna. Médico Jefe del Programa de Salud del Adulto y Asesor Sub-Programas de Hipertensos.
} 


\section{Introducción}

La hipertensión arterial constituye una amenaza silenciosa para la salud del hombre y un verdadero desafío para la atención en salud, dado que un incremento en la presión arterial sistémica aumenta el riesgo de accidentes vasculares cerebrales y cardiopatía coronaria; constituyendo una condición que debe prevenirse y tratarse.

En Chile, la hipertensión es un problema de salud prioritario por su alta prevalencia una condición que debe prevenirse y tratarse.

En Chile, la hipertensión arterial es un problema de salud prioritario por su alta prevalecía en la población adulta. El tratamiento de la hipertensión arterial de asocia con una reducción significativa de la morbimortalidad cardiovascular, primera causa de muerte en nuestro país. Según estudios proyectivos del Departamento de Planificación del Ministerio de Salud de Chile, se perfila que las enfermedades cardiovasculares representarán la primera causa de muerte en el año 2000 , con un $31,8 \%$ del total de defunciones ${ }^{1}$.

La Organización Mundial de la Salud (OMS) y diversos países, conscientes del problema han impulsado medidas orientadas a prevenir o reducir las complicaciones asociadas a la hipertensión arterial y a otras enfermedades cardiovasculares. La OMS, a través de su comité de expertos, recomienda entre sus medidas incorporar a la atención de salud estrategias no farmacológicas que por sí solas o como apoyo a la terapia tradicional contribuyan a reducir la alta marbimortalidad evidenciada en los diversos países.

El marco teórico que sustenta el presente estudio lo constituyen:

a) El modelo en creencias en salud expandido hacia el rol en enfermo de M. Becker, el cual entrega un marco de referencia a la decisión que tornará el sujeto para adherirse a un tratamiento y cambiar conductas en salud. Explica como las propias percepciones de las personas influencian las conductas que adoptarán en beneficio de su salud. Estos beneficios deben ser mayores que el costo social. Emocional, económico y biológico para que esta persona decida adherirse a un programa. La motivación juega un rol relevante en este modelo, ya que tiene una doble dimensión: el estado psicológico de disposición para realizar acciones específicas y la creencia que un curso particular de acción será beneficiosa en la reducción de la amenaza. 


\section{EL MODELO DE CREENCIAS EN SALUD Y CONDUCTAS DEL ROL DE ENFERMO}

\author{
ASPECTOS RECOMENDADOS A \\ CONTROLAR EN LA CONDUCTA \\ DEL ROL DE ENFERMO
}

FACTORES MODIFICANTES Y

HABILITANTES
CONDUTAS DEL ROL DE

ENFERMO

\begin{abstract}
Demográficos (muy joven o anciano)
Estructurales (costo, duración, complejidad, efectos secundarios, accesibilidad al régimen, Necesidad de nuevos patrones de conductas).
\end{abstract}

Valor de la reducción de la amenaza

\section{de enfermedad}

- $\quad$ Susceptibilidad o resuceptibilidad

(incluye creencia en el diagnóstico).

- Vulnerabilidad a enfermedades en

general

Extensión de posible daño corporal

Extensión de posible interferencia

con roles sociales.

Presencia de (o experiancia pasada)

síntomas.
Actividades: (Satisfacción con la visita al personal de salud con los procedimientos clínicos y facilidades.

Interacción: (duración, profundidad,

continuidad, expectación mutua, calidad y tipo de interrelación, acuerdo del personal de salud con el paciente, retroalimentación con el paciente)

Habilitantes: (previa experiencia en la Acción, la enfermedad o el régimen, fuentes de consejo o referencias).

\section{Probabilidad de: \\ - Adherencia de regímes prescritos \\ (VG: drogas, dietas, ejercicios, hábitos personales y de trabajo, test de seguimiento, entrevista de seguimiento, entrar o continuar un programa de tratamiento).}

\section{Probalidad que la conducta}

adherence reducirá la amenaza

Estimación subjetiva de:

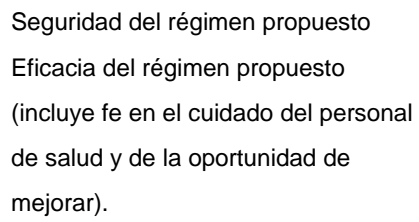

b) Conceptos teóricos de la teoría de aprendizaje de los neoconductistas, donde la modificación de hábitos y de estilos de vida es un proceso de reforzamiento de un operante, su diferenciación y los procesos relacionados con ello. El cambio se logra mediante la práctica y en una interacción entre los individuos y sus ambientes proporcionando los estímulos adecuados en el momento 
oportuno. El neoconductivismo ve al hombre como una combinación de su herencia genética y las experiencias adquiridas a través de su interacción com su ambiente. El ser humano actúa con propósitos, como una consecuencia de haber recibido refuerzos pasados, los cuales lo orienta hacia la repetición del acto para recibir los mismos refuerzos en el futuro. Así se puede decir que la persona está motivada porque tiene expectativas.

Al interrelacionar a y $b$, podemos decir que:

El paciente hipertenso severo se siente motivado, acepta las direcciones del personal de salud y manifiesta su intención de adherirse al programa, cuando percibe que la consejería personalizada en un tratamiento seguro, sin efectos secundarios, sin costo agregado, de fácil acceso y que se vislumbra eficaz al disminuir la sintomatología presentada, así como también reducir la probabilidad de complicaciones asociadas como accidente cerebro vascular, cardiopatía coronaria y mantener cifras tensionales en rangos normales o cercamos a la normalidad. El paciente percibe así una reducción significativa de su amenaza.

Durante las sesiones de Consejería Personalizada donde se trata a la persona en forma integral y no sólo a su enfermedad, se refuerza al paciente en forma positiva cuando ha emitido durante un tiempo conductas esperadas propuestas conjuntamente en sesiones anteriores. Estos refuerzos hacen que la persona repita las conductas que queremos lograr como una consecuencia de haber recibido los refuerzos pasados. Decimos entonces que la persona está motivada ya que el paciente se encuentra en un estado de disposición para realizar acciones específicas que esperamos que él logre.

El paciente parte motivado por las perspectivas que le ofrece la Consejería Personalizada de reducir la amenaza y permanece continuamente motivado por el refuerzo positivo ante conductas esperadas; lo que trae como consecuencia una mayor probabilidad de adherencia al programa y de lograr cambios conductuales esperados; evidenciándose en una reducción de cifras tensionales sistólicas y diastólicas y el aumento de su autoestima.

A medida que aumenta la autoestima del paciente y reduce sus cifras tensionales, estos actúan a su vez como refuerzos positivos, lo que hace que el paciente presente una mejor disposición psicológica a actuar y una mayor probabilidad de que repita las conductas esperadas y que éstas permanezcan en el tiempo. 
Paciente hipertenso

severo motivado por

actúa como refuerzo +

la percepción de la

reducción de la

amenaza y estimación

CONSEJERÍA

ADHERENCIA

T.A.

de seguridad y

PERSONALIZADA

PROGRAMA

AUTOESTIMA

eficacia del

tratamiento

propuesto

actúa como refuerzo +

La presente investigación formula y introduce un método de intervención no farmacológico la "Consejería Personalizada" como un método de intervención de Enfermería, donde se interactúa con el paciente en un plano que va más allá de la problemática de la enfermedad. Se centra en la persona con sus particularidades e intereses, estableciéndose un rapport que permite el trabajo en un ambiente terapéutico.

La Consejería Personalizada, variable independiente de este estudio, contempla diversas actividades:

01. Valoración del ambiente físico, social y privado del individuo, obteniendo la mayor información posible, detectando con especial énfasis los conocimientos que tenga o no en relación a su enfermedad.

02. Diagnóstico de Enfermería, destacando aquellas conductas que se relacionan con la modificación de estilos de vida que se asocian a la hipertensión arterial y las necesidades percibidas como prioritarias por los pacientes. Una vez realizado el diagnóstico se planifica el programa educativo, que se desarrolla en las diversas sesiones de trabajo.

03. Ejecución o Intervención, se proponen metas en conjunto alcanzables para el paciente y así éste asume la responsabilidad de sus propios cambios conductuales. Se le orienta para facilitar que logre los cambios esperados a la vez de promover conductas de auto cuidado. Se educa en relación a sus necesidades. Se refuerza positivamente la elicitación de conductas esperadas. Se escucha atentamente al paciente ya que es una de las mejores estrategias del proceso de ayuda incidiendo enormemente junto a las demás actividades en la motivación y autoestima de la persona.

04. Evaluación permanente de cambios conductuales para retroalimentar el proceso de Intervención de Enfermería.

Diversas investigaciones, OGANOV. R. G. et al. ${ }^{7}$; J. M., JALOWIEC, A. ${ }^{10}$; FASCE, E. et al. ${ }^{2}$; GUTZWILLER, F. ${ }^{4,5}$; ROCCELA, E. et al. ${ }^{11}$; HORAN, M. ROCCELA, E. ${ }^{6}$, han encontrado que algunas 
medidas no farmacológicas han producido un impacto en la prevención como en la intervención de la hipertensión arterial y sus complicaciones.

FLORENZANO, R. et al. $^{3}$ encontraron resultados más positivos en pacientes en que la atención fue más personalizada, señalando que la educación es un factor importante en el éxito del tratamiento y que el profesional de enfermería es el agente que puede obtener los resultados anteriormente descritos.

OWEN, L. A. et al. ${ }^{8}$ y TOCHIKUBO, O. et al. ${ }^{12}$ destacan la importancia de la educación y de la consejería, avalando que estas estrategias no farmacológicas tienen importancia en la prevención e intervención de pacientes con hipertensión arterial esencial.

PRAVIC, T. ${ }^{9}$, utilizó la estrategia consejería personalizada para modificar estilos de vida en estudiantes universitarios obesos, logrando significancía estadística en la modificación de hábitos dietéticos. Este método de intervención tuvo una alta adherencia, por lo que la autora la recomienda como una estrategia para tratar a pacientes obesos y sugiere sea aplicada en pacientes con enfermedades de carácter crónico.

El propósito de la presente investigación fue: Evaluar los efectos de un Programa de Consejería Personalizada orientado a reducir cifras tencionales y aumentar autoestima en pacientes hipertensos esenciales severos OMS I, adscritos al programa de Hipertensión Arterial del Hospital Las Higueras de Talcahuano-Chile.

Se plantearon además, los siguientes objetivos:

01. Conocer variables sociodemográficas asociadas a los pacientes hipertensos esenciales severos OMS I, atendidos en Policlínico de Hipertensão Arterial, Hospital Las Higueras, Talcahuano-Chile. Las variables estudiadas fueran: sexo, edad, estado civil, educación formal, ingreso familiar, religión.

02. Conocer estilo de vida asociado a pacientes hipertensos esenciales severo OMS I atendidos en Policlínico de Hipertensão Arterial, Hospital Las Higueras, Talcahuano-Chile. Se seleccionan las siguientes variables: hábito de ejercicio, mitos y creencias en relación a su enfermedad, grado de conocimiento de su enfermedad, ingesta de alcohol manifestada por los pacientes, percepción del paciente de la dinámica de su grupo familiar, antecedentes hipertensión arterial familiar declarados por el paciente.

03. Conocer presencia o ausencia de dependencia entre peso y presión arterial sistólica y entre peso y presión arterial diastólica en pacientes hipertensos esenciales severos OMS I, atendidos en en Policlínico de Hipertensão Arterial, Hospital Las Higueras, Talcahuano-Chile.

Se trabajaron las siguientes hipótesis:

Pacientes hipertensos esenciales severos OMS I, que se adscriben a un programa de 
consejería personalizada, disminuyen su presión arterial sistólica en promedio más que pacientes del grupo control.

Pacientes hipertensos esenciales severos OMS I, que se adscriben a un programa de consejería personalizada, disminuyen su presión arterial diastólica en promedio más que consejería personalizada, aumentan su autoestima en promedio más que pacientes del grupo control.

\section{Materiales y metodos}

El universo estuvo constituido por 45 pacientes adultos hipertensos esenciales que a junio de 1990 tenían una permanencia de a lo menos 6 meses se el Programa de Hipertensión Arterial de Policlínico de Hipertensión del Hospital Las Higueras, Talcahuano-Chile.

Para conformar la muestra y cuidar su validez interna se estimó controlar las variables: edad, tipo de hipertensión arterial, tipo de tratamiento medicamentoso, tiempo de permanencia en el programa, cifras tensionales de ingreso, potologias asociadas y consentimiento del paciente.

La muestra quedó constituida por 23 pacientes que cumplían con los requisitos estipulados en las variables de control, quedando conformada por: pacientes adultos, con hipertensión arterial severa OMS I, tratamiento médico individualizado y que a junio 1990 tenían una permanencia de a lo menos 24 semanas en el Programa de Hipertensión Arterial de Policlínico de Hipertensión del Hospital Las Higueras de Talcahuano-Chile, manteniendo cifras tensionales compatibles con la categoría de hipertensión arterial severa sin outra patología que agravan su condición de hipertenso y que accedieron a ser incorporados al estudio.

Conformada la muestra; los grupos de trabajo se constituyeron utilizando para esse efecto la tabla de números aleatorios incorporándose 10 pacientes al grupo control y 13 al experimental.

Los pacientes del grupo control recibieron la atención normada en el servicio con un control médico mensual u su terapia medicamentosa individualizada. El grupo experimental fuera de recibir la atención normada se incorporó a un Programa de Consejería Personalizada, cuya duración fue de 24 semanas, con 2 sesiones de trabajo al mes. En ambos grupos de trabajo, experimental y control se mantuvo la terapia medicamentosa inicial durante el período que duró el programa de intervención.

Para llevar a cabo este trabajo se seleccionó el diseño pre-test, post-test con grupo control. 


\section{Diseño Experimental}

Grupo Control

\section{Grupo Experimental}

Pre-Test

Escala autoestima

Control presión arterial

Control peso

Tratamiento

Programa de consejería personalizada, cuya duración fue de 24 semanas, com 2 sesiones de trabajo mensual con un promedio de 40 minutos por paciente

Pre-Test

Escala autoestima

Control presión arterial

Control peso

\author{
Escala autoestima \\ Control presión arterial \\ Control peso
}

Las variables dependientes de este estudio, presión arterial sistólica y diastólica se midieron con un esfigmomanómetro mercurial y su control se realizó a través de normas internacionales, y la autoestima a través de la escala de autoestima de Tennessee desarrollada por Wiliams $\mathrm{H}$. Fitts; que entrega una visión bastante amplia de la autoestima de las personas, ya que considera los aspectos del yo físico, yo moral, yo personal, yo familiar, y el yo social; al mismo tiempo que muestra la dinámica asociada a cada uno de ellos, el modo como la persona se percibe, el modo como la persona se percibe, el modo como la personal acepta o rechaza a sí mismo.

La escala está constituida por cien enunciados descriptivos lo que permite obtener un retrato que la persona hace de sí mismo.

Para conocer presencia o ausencia de dependencia entre peso y presión arterial sistólicay diastólica se calculó la covarianza, considerando la globalidad de los pacientes que finalizaron el estudio; $(n=19)$.

Para trabajar las hipótesis planteadas se utilizó la dócima: diferencias de medias con varianzas poblacionales desconocidas e iguales, teniendo como supuesto que las poblaciones en estudio tienen una distribución normal.

Las hipótesis planteadas se estructuran de la siguiente forma:

Hipótesis de trabajo (H.T.): $\mu_{1}>\mu_{2}<--->\mu_{1}-\mu_{2}>0$

Contrahipotesis (C.H.): $\mu_{1}<\mu_{2}<--->\mu_{1}-\mu_{2}<0$ 
Estadísticamente se precedió a calcular el valor t utilizando t student con un $\alpha$. Los resultados de la prueba deben interpretarse de la siguiente manera: si $t_{c}>t_{0,05}\left(n_{1}+n_{2}-2\right)$, se acepta la Hipótesis de Trabajo, en caso contrario se rechaza.

\section{Resultados}

\section{Descripción de los participantes del estudio}

Grupo control: El 71,4\% de los pacientes son del sexo masculino, siendo su rango de edad entre 46 a 65 años. El 57,1\% son casados y el 100\% de ellos no completaron se educación básica. El ingreso familiar es igual o inferior a \$36.000 (100 dólares aproximadamente al momento del estudio), en el $71,4 \%$ de los pacientes. Solamente el 14,3\% declaran no tener religión; siendo la católica o evangélica la profesada por el resto de los pacientes. Los pacientes adscritos al grupo control en un $71,4 \%$ realizaban actividad física regular. El $100 \%$ de ellos tienen incorporado algún mito o creencia en torno a su enfermedad, como también no tienen o tienen escaso conocimiento y manifestaron ser abstemios. La totalidad de los pacientes de este grupo perciben como buena la dinámica de su grupo familiar y el 85,7\% desconoce antecedentes familiares de hipertensión arterial.

Grupo experimental: Poco más de la mitad de los pacientes son del sexo femenino, siendo el rango de edad entre 46 a 65 años en el 91,7\% de los pacientes. El 58,4\% son casados y en lo que respecta a la variable educación formal el 16,7\% no recibió educación, el $58,3 \%$ no completó su enseñanza básica y el $25 \%$ iniciaron estudios en la enseñanza media. Su ingreso familiar en el $83,3 \%$ de los pacientes es igual o inferior a $\$ 36.000$, en igual porcentaje al anterior manifestaron profesar una religión. El 25\% de los pacientes del grupo experimental realiza ejercicios regularmente y en igual porcentaje percibe su dinámica familiar como mala. El 33,3\% tiene algún mito o creencia de su enfermedad y la totalidad no tiene o tiene escaso conocimiento de ella. En relación a ingesta alcohólica, el 8,3\% es alcohólico, en el $16,7 \%$ su ingesta es moderada y el $75 \%$ es abstemio. El $91,7 \%$ de los pacientes desconoce tener antecedentes familiares de hipertensión arterial.

- Dependencia entre variables

Se constató una dependencia directa entre peso y presión sistólica $(\operatorname{cov}(\mathrm{x}, \mathrm{y})=$ $25,17)$ y entre peso y presión arterial diastólica $(\operatorname{cov}(x, y)=11,76)$. Siendo las variables presión arterial sistólica y presión diastólica directamente dependiente, es decir un aumento en el peso corporal provoca un incremento en la presión arterial sistólica y diastólica de los pacientes. 


\section{- Hipótesis}

Para la variable dependiente presión arterial sistólica, la disminución promedio de la presión arterial sistólica en pacientes del grupo experimental fue significativamente mayor en relación a pacientes del grupo control.

Grupo experimental: $X=37,833 ; \mathrm{DS}=34,40$. Grupo control $X=16,857 ; \mathrm{DS}=49,21 . \mathrm{t}_{\mathrm{c}}(17)=$ 1,$030 ; p>0.05$.

La disminución promedio de la presión arterial diastólica en pacientes del grupo experimental fue mayor al pesquisado en pacientes del grupo control.

Grupo experimental: $X=24,58$; $D S=14,78$. Grupo control $X=12,0 ; D S=22,16 . t_{c}(17)=$ 3,$522 ; p>0.05$.

Para la variable dependiente autoestima se constató que los pacientes adscritos al programa de consejería personalizada presentaron un incremento mayor en su autoestima que pacientes del grupo control.

Grupo experimental: $X=4,33 ; \mathrm{DS}=25,914$. Grupo control $X=-12,43 ; \mathrm{DS}=18,25 . \mathrm{t}_{\mathrm{c}}(17)=$ 0,$085 ; p>0.05$.

\section{Conclusiones y Discusión}

Los hallazgos del presente trabajo son compatibles con conclusiones de estudios nacionales y extranjeros que proponen implementar métodos de intervención no farmacológicos, como es la conserjería personalizada en la atención de pacientes hipertensos, demostrando su eficacia en la reducción de cifras tensionales y de otros factores de riesgo asociados a la hipertención arterial en el grupo estudiado.

Se pudo constatar que pacientes hipertensos esenciales severos OMS I, adscritos al Programa de Consejería Personalizada del Hospital las Higueras, Talcahuano-Chile, se adhirieron más al programa; teniendo como resultado final una disminución de cifras tencionales y un mayor incremento en su autoestima que pacientes del grupo control.

Fue posible observar presencia de dependencia entre peso y presión arterial; una reducción en el peso de los pacientes hipertensos esenciales severos OMS I, provoca una disminución en la presión arterial sistólica y/o diastólica de las personas.

Los resultados de la presente investigación, así como de otras permiten ratificar a la Consejería Personalizada como una buena estrategia, debiendo incorporarse en la enseñanza como un método de intervención de Enfermería, en especial para lograr cambios conductuales en personas con enfermedades crónicas.

Se estima importante continuar con esta línea de investigación, proponiendo se 
estudie la Consejería Personalizada como unidad de intervención, en pacientes con características sociodemográficas diferentes a las del presente trabajo, en personas con diferentes patologías especialmente de carácter crónico; como también se hace necesario evaluar su efecto a través del tiempo.

La escala de autoestima utilizada en este trabajo, validada por diversos autores y recomendada para ser aplicada en personas mayores de 12 años; constituye una de las limitantes en el desarrollo del presente trabajo, pesquisándose en su aplicación dificultad en la comprención de algunas de las preguntas del cuestionario por parte de los pacientes de la muestra, dado su bajo nivel instruccional a juicio de los autores del presente trabajo.

Two intervention systems to evaluate the effect of personalized counseling in severe essential hypertensive patients OMS I, oriented to reduce systolic and diastolic tensional pressures and to increase selfesteem, were compared. The independent variable as an intervention method took place during 24 weeks. Dependent variables: tensional pressures and self-esteem were measured. The results showed that essential severe hypertensive patients OMS I of the experimental groups decreased the average of (the systolic and diastolic) tensional pressures and increased their self-esteem more than patients of control group. Finding also a direct dependence between weight and tensional pressures Personalized Counseling showed to be a successful strategy of nursing for this group of patients.

UNITERMS: Counseling, personalized counseling, hipertense patient.

\section{REFERENCIAS BIBLIOGRÁFICAS}

01. CHILE. Ministerio de Salud. Departamento de Planificación. Proyecciones de la Mortalidad y Morbilidad. Chile, 1982.

02. FASCE, E. et al. Efectos de la supreción de un programa especial de control y tratamiento de pacientes hipertensivos. Bol. Cardiol., Chile, v. 5, n. 1, p. 27-39, 1986.

03. FLORENZANO, R. et al. Permanencia en tratamiento antihipertensivo: comparación de dos sistemas de seguimiento. Bol. Of. Sanit. Panam., XCL, n. 5, p. 428-40, 1981. 
04. GUTSWILLER, G. Issues in health education: epidemiology and prevention of hypertension in Switzerland. Prev. Med., 14, n. 4, p. 532-40, 1985.

05. . Health education and public health polices for detection, prevention and treatment of hypertension. J. Clin. Hypertension, v. 2, n. 2, p. 210-5, 1986.

06. HORAN, M., ROCCELA, E. Nonpharmacologic treatment of hypertension: does it work? Eur. Heart J., v. 8, p. 77-86, 1987. Suppl. B.

07. OGANOV, R. G. et al. Se intensifica la lucha contra la hipertensión esencial. Foro Mundial de la Salud, v. 9, p. 90-3, 1983.

08. OWEN, L. A. et al. Counseling patients about diet and nutrition. Nurs. Clin. North Am., v. 14, n. 2, p. 247-68, 1979.

09. PARAVIC, T. Aplicación de un programa de enfermería integral en adolescentes universitarios obesos para reducir de peso. Quito: ALADEFE - Asociación Latinoamericana de Escuelas y Faculdades de Enfermería, 1990. V. 1, p. 46-8.

10. POWERS, J. M., JALOWIEC, A. Profile of the well-controlled, well adjusted hypertensive patient. Nurs. Res., v. 36 n. 2, p. 106-10, march/april, 1987.

11. ROCCELA, E. et al. Hypertension knowledge, attitudes and behavior. Public Health Rep., v. 101, n. 6, p. 599-606, 1986.

12. TOCHIKUBO, O. et al. Management of hypertension in high school by using new salt titrator tape. Hypertension, v. 8, n. 12, p. 1164-71, 1986. 